\title{
Negative Ion Photoelectron Spectroscopy Confirms the Prediction of the Relative Energies of the Low-Lying Electronic States of 2,7- Napthoquinone
}

\author{
Zheng Yang, ${ }^{\S}$ David A. Hrovat, ${ }^{\dagger}$ Gao-Lei Hou, ${ }^{\S}$ Weston Thatcher Borden ${ }^{* \dagger}$, and Xue-Bin \\ Wang $^{* \S}$ \\ ${ }^{\S}$ Physical Sciences Division, Pacific Northwest National Laboratory, P. O. Box 999, MS K8-88, \\ Richland, WA 99352, USA \\ ${ }^{\dagger}$ Department of Chemistry and the Center for Advanced, Scientific Computing and Modeling, \\ University of North Texas, 1155 Union Circle, \#305070, Denton, Texas 76203-5070, USA
}

\author{
Corresponding Authors \\ Weston T. Borden: Weston.Borden@,unt.edu \\ Xue-Bin Wang: Xuebin.wang@pnnl.gov
}




\begin{abstract}
Cryogenic negative ion photoelectron (NIPE) spectra of the radical anion of 2,7napthoquinone $\left(\mathbf{N Q}^{\bullet}\right)$ ) have been taken at $20 \mathrm{~K}$, using 193, 240, 266, 300, and $355 \mathrm{~nm}$ lasers for electron detachment. The electron affinity of the NQ diradical is determined from the first resolved peak in the NIPE spectrum to be $2.880 \pm 0.010 \mathrm{eV}$. CASPT2/aug-cc-pVDZ calculations predict with reasonable accuracy the positions of the $0-0$ bands in the three lowest electronic states of NQ. In addition, the Franck-Condon factors calculated from the CASPT2/aug-cc-pVDZ optimized geometries, vibrational frequencies, and normal modes, successfully simulate the vibrational structures in these bands. The NIPE spectrum of $\mathbf{N Q}^{\bullet-}$ confirms that, as predicted, ${ }^{3} \mathrm{~B}_{2}$ is the ground state, and the ${ }^{1} \mathrm{~B}_{2}$ and ${ }^{1} \mathrm{~A}_{1}$ states are, respectively, 12.7 and $16.4 \mathrm{kcal} / \mathrm{mol}$ higher in energy than the triplet ground state. The experimental value of $\Delta E_{\mathrm{ST}}=12.7 \mathrm{kcal} / \mathrm{mol}$ in $\mathbf{N Q}$ and the finding that ${ }^{1} \mathrm{~B}_{2}$ is the lower energy of the two singlet states confirm the results of the previous calculations on NQ. These calculations predicted an increase in $\Delta E_{\mathrm{ST}}$ on the substitution of both methylene groups in 2,7-naphthoquinodimethane (NQDM) by oxygens in NQ, thus providing a dramatic contrast to the decrease of $17.5 \mathrm{kcal} / \mathrm{mol}$ in $\Delta E_{\mathrm{ST}}$ found for substitution of one methylene group by one oxygen on going from trimethylenemethane (TMM) to oxyallyl (OXA).
\end{abstract}




\section{Introduction}

The greater strength of the $\mathrm{C}=\mathrm{O} \pi$ bond in the oxyallyl (OXA) diradical, relative to a $\mathrm{C}=\mathrm{C} \pi$ bond in the trimethylenemethane (TMM) diradical, and the ability of the $\mathrm{C}=\mathrm{O} \pi *$ orbital in OXA to accept an electron pair were predicted to result in a very large reduction in the singlettriplet energy separation $\left(\Delta E_{\mathrm{ST}}\right)$ on going from TMM to OXA. ${ }^{1,2}$ Subsequently, Lineberger and coworkers, used negative ion photoelectron spectroscopy (NIPES) ${ }^{3,4}$ to confirm this prediction. As shown in Figure 1, NIPES found a decrease of $17.5 \mathrm{kcal} / \mathrm{mol}$ in $\Delta E_{\mathrm{ST}}$ between $\mathbf{T M M}\left(\Delta E_{\mathrm{ST}}=\right.$ $16.2 \mathrm{kcal} / \mathrm{mol}$, triplet ground state $)^{5,6}$ and $\mathbf{O X A}\left(\Delta E_{\mathrm{ST}}=-1.3 \mathrm{kcal} / \mathrm{mol}\right.$, singlet ground state) ${ }^{7,8}$

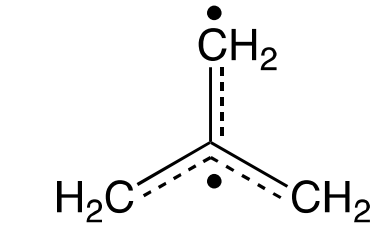

TMM

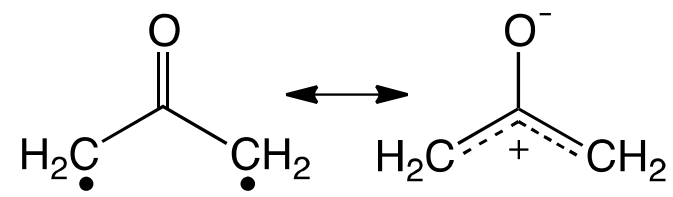

OXA

$$
\Delta E_{\mathrm{ST}}=16.2 \mathrm{kcal} / \mathrm{mol} \quad \Delta E_{\mathrm{ST}}=-1.3 \mathrm{kcal} / \mathrm{mol}
$$

Figure 1. Singlet-triplet energy separations $\left(\Delta E_{\mathrm{ST}}\right)$ in trimethylenemethane (TMM) $)^{5,6}$ and oxyallyl (OXA), ${ }^{7,8}$ measured by negative ion photoelectron spectroscopy (NIPES). ${ }^{3,4}$

Based on these results, it might have been expected that a similarly large decrease in $\Delta E_{\mathrm{ST}}$ should occur on replacement of the two methylene groups in the metabenzoquinodimethane (MBQDM) diradical with the two oxygens in the meta-benzoquinone (MBQ) diradical. However, electronic structure calculations predicted only a very small decrease in $\Delta E_{\mathrm{ST} .} \cdot{ }^{910}$ This prediction was confirmed experimentally, two decades later, when, as shown in Figure 2, NIPES found the value of $\Delta E_{\mathrm{ST}}$ in $\mathbf{M B Q}\left(\Delta E_{\mathrm{ST}}=9.0 \mathrm{kcal} / \mathrm{mol}\right)^{10,11}$ to be only $0.6 \mathrm{kcal} / \mathrm{mol} \mathrm{smaller} \mathrm{than} \mathrm{that} \mathrm{in} \mathbf{M B Q D M}\left(\Delta E_{\mathrm{ST}}=9.6 \mathrm{kcal} / \mathrm{mol}\right){ }^{12}$ 


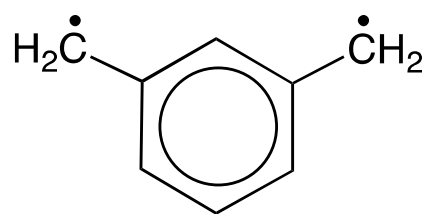

MBQDM ( $\left.{ }^{1} A_{1}\right)$

$\Delta E_{\mathrm{ST}}=9.6 \mathrm{kcal} / \mathrm{mol}$<smiles>O=C1C=CC(=O)C=C1</smiles>

$M B Q\left({ }^{1} B_{2}\right)$

$\Delta E_{\mathrm{ST}}=9.0 \mathrm{kcal} / \mathrm{mol}$

Figure 2. $\Delta E_{\mathrm{ST}}$, measured by NIPES, ${ }^{3,4}$ between the triplet ground state $\left({ }^{3} \mathrm{~B}_{2}\right)$ and the lowest singlet state, ${ }^{1} \mathrm{~A}_{1}$ in meta-benzoquinodimethane (MBQDM) ${ }^{12}$ and ${ }^{1} \mathrm{~B}_{2}$ in meta-benzoquinone (MBQ). ${ }^{10,11}$

The reason for the very small change in the size of $\Delta E_{\mathrm{ST}}$ on going from MBQDM to MBQ was given in the theoretical paper that predicted this change would, in fact, be found to be small. ${ }^{9,10}$ In MBQDM the lowest singlet state is ${ }^{1} \mathrm{~A}_{1}$, in which the aromaticity of the benzene ring is largely preserved by localizing the two nonbonding electrons on the exocyclic methylene groups. However, in $\mathbf{M B Q}$ the lowest singlet state is ${ }^{1} \mathrm{~B}_{2}$, due to the strength of $\pi$ bonds to the carbonyl groups in this state. The ${ }^{1} \mathrm{~B}_{2}$ state is stabilized, relative to the ${ }^{3} \mathrm{~B}_{2}$ ground state, by $\geq 12.5$ $\mathrm{kcal} / \mathrm{mol}$ on going from $\mathbf{M B Q D M} \mathbf{M}^{12}$ to $\mathbf{M B Q} .^{10,11}$

However, $\Delta E_{\mathrm{ST}}$ is measured between the triplet and the lowest singlet state, which changes from ${ }^{1} \mathrm{~A}_{1}$ in $\mathbf{M B Q D M}$ to ${ }^{1} \mathrm{~B}_{2}$ in $\mathbf{M B Q}$. Since ${ }^{1} \mathrm{~B}_{2}$ starts off $\geq 11.9 \mathrm{kcal} / \mathrm{mol}$ above the lowest singlet state $\left({ }^{1} \mathrm{~A}_{1}\right)$ in $\mathbf{M B Q D M},{ }^{12}$ the $\geq 12.5 \mathrm{kcal} / \mathrm{mol}$ stabilization of ${ }^{1} \mathrm{~B}_{2}$, relative to ${ }^{3} \mathrm{~B}_{2}$, in $\mathrm{MBQ}$ results in a decrease in $\Delta E_{\mathrm{ST}}$ of only about $0.6 \mathrm{kcal} / \mathrm{mol}$.

Very recently, calculations have predicted a similar change in the nature of the lowest singlet state in going from the 2,7-napthoquinodimethane (NQDM) diradical to the 2,7napthoquinone (NQ) diradical. ${ }^{13}$ However, in this case the ${ }^{1} \mathrm{~B}_{2}$ state is calculated to be 28.3 $\mathrm{kcal} / \mathrm{mol}$ above the ${ }^{3} \mathrm{~B}_{2}$ ground state in NQDM; whereas, the ${ }^{1} \mathrm{~A}_{1}$ state of NQDM is computed to 
be only $5.5 \mathrm{kcal} / \mathrm{mol}$ above the ${ }^{3} \mathrm{~B}_{2}$ ground state. Consequently, although the ${ }^{1} \mathrm{~B}_{2}$ state is calculated to be stabilized, relative to ${ }^{3} \mathrm{~B}_{2}$, by $17.3 \mathrm{kcal} / \mathrm{mol}$ on going from NQDM to NQ, Figure 3 shows that the value of $\Delta E_{\mathrm{ST}}$ is actually predicted to increase from $\Delta E_{\mathrm{ST}}=5.5 \mathrm{kcal} / \mathrm{mol}$ in

NQDM to $\Delta E_{\mathrm{ST}}=11.0 \mathrm{kcal} / \mathrm{mol}$ in $\mathbf{N Q}$.

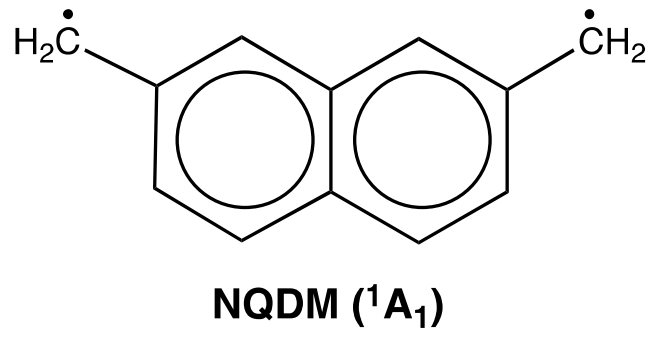

$\Delta E_{\mathrm{ST}}=5.5 \mathrm{kcal} / \mathrm{mol}$

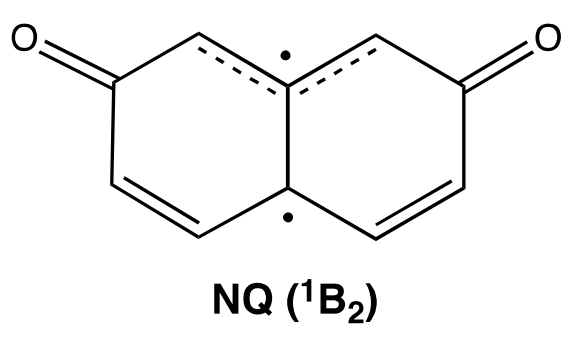

$\Delta E_{\mathrm{ST}}=11.0 \mathrm{kcal} / \mathrm{mol}$

Figure 3. $\Delta E_{\mathrm{ST}}$, calculated between the triplet ground state $\left({ }^{3} \mathrm{~B}_{2}\right)$ and the lowest singlet state, ${ }^{1} \mathrm{~A}_{1}$ in 2,7-naphthoquinodimethane (NQDM) and ${ }^{1} \mathrm{~B}_{2}$ in 2,7-naphthoquinone (NQ). ${ }^{13}$

This predicted increase of $5.5 \mathrm{kcal} / \mathrm{mol}$ in $\Delta E_{\mathrm{ST}}$ on the substitution of both methylene groups in NQDM by oxygens in NQ, provides a dramatic contrast to the decrease of 17.5 $\mathrm{kcal} / \mathrm{mol}$ in $\Delta E_{\mathrm{ST}}$ found experimentally for TMM $\rightarrow$ OXA. As a first step in testing this prediction for NQDM $\rightarrow$ NQ, we have obtained the NIPE spectrum of $\mathbf{N Q}^{\bullet}$, a distonic radical anion ${ }^{14,15}$ with potentially important applications in a variety of contexts. A careful analysis of the vibrational structure of the peaks that correspond to the two lowest singlet states of NQ confirms that, as predicted, ${ }^{13}$ the energy of the ${ }^{1} \mathrm{~B}_{2}$ state is below that of the ${ }^{1} \mathrm{~A}_{1}$ state. NIPES provides an experimental value of $\Delta E_{\mathrm{ST}}=12.7 \mathrm{kcal} / \mathrm{mol}$ in $\mathbf{N Q}$, which is about $2 \mathrm{kcal} / \mathrm{mol}$ larger than the calculated value of $\Delta E_{\mathrm{ST}}=11.0 \mathrm{kcal} / \mathrm{mol}^{13}$

\section{Experimental Methodology}


The negative ion photoelectron spectroscopy (NIPES) experiments were carried out at PNNL using an apparatus consisting of an electrospray ionization (ESI) source, a temperature controlled cryogenic ion trap, and a magnetic-bottle time-of-flight (TOF) photoelectron spectrometer. ${ }^{16}$ A $0.1 \mathrm{mM}$ acetonitrile solution of 2,7-dihydroxynaphalene, titrated with a small amount of $\mathrm{NaOH}$ dissolved in water, was prepared in a $\mathrm{N}_{2}$ glovebox, and used to generate $\mathbf{N Q}^{\mathbf{-}}$ by electrospray under an $\mathrm{N}_{2}$ atmosphere. The inert $\mathrm{N}_{2}$ environment helps reduce unwanted radical reactions in solution and in the sprayed droplets, thus helping to generate strong and stable ion beams. The ESI conditions were optimized to ensure that the doubly deprotonated species, $\mathbf{N Q}^{--}$, was a prominent peak in the mass spectrum. The ions generated by ESI were guided by quadrupole ion guides into the ion trap, where they were accumulated and cooled for $20-100 \mathrm{~ms}$ by collisions with cold buffer gas $\left(20 \% \mathrm{H}_{2}\right.$ balanced in helium) at $20 \mathrm{~K}$, before being transferred into the extraction zone of a TOF mass spectrometer. The cooling of the anions to $20 \mathrm{~K}$ improved the spectral energy resolution and minimized the possibility of the appearance of peaks in the NIPE spectra resulting from hot bands (see Supporting Information, as well as Ref.16).

The $\mathbf{N Q}^{\mathbf{-}}$ ions were then mass selected and maximally decelerated before being photodetached. In the current study, photon energies of $193 \mathrm{~nm}(6.424 \mathrm{eV})$ from an excimer laser, $266 \mathrm{~nm}(4.661 \mathrm{eV})$ and $355 \mathrm{~nm}(3.496 \mathrm{eV})$ from a Nd:YAG laser, and $240 \mathrm{~nm}(5.166 \mathrm{eV})$ and $300 \mathrm{~nm}(4.133 \mathrm{eV})$ from doubling frequency of $480 \mathrm{~nm}$ and $600 \mathrm{~nm}$ photons from an OPO/OPA laser were used. The lasers were operated at a $20 \mathrm{~Hz}$ repetition rate, with the ion beam off at alternating laser shots, in order to enable shot-to-shot background subtraction. Photoelectrons were collected with ca. 100\% efficiency with the magnetic bottle and analyzed in a $5.2 \mathrm{~m}$ long electron-flight tube. The recorded TOF photoelectron spectrum was converted into 
an electron kinetic-energy spectrum by calibration with the known NIPE spectra of $\mathrm{I}^{-17}{ }^{17} \mathrm{Au}^{-18},{ }^{18}$ $\mathrm{OsCl}_{6}{ }^{2-},{ }^{19}$ and thiophenoxide. ${ }^{20}$ The electron-binding energy (EBE) was obtained by subtracting the electron kinetic energy from the energy of the detaching photons. The energy resolution was about $2 \%$ (i.e., $\sim 20 \mathrm{meV}$ for $1 \mathrm{eV}$ kinetic-energy electrons).

\section{Computational Methodology}

Geometries were optimized and vibrational analyses were performed with $(12 / 12)$ CASSCF calculations, in which all twelve of the $\pi$ electrons were correlated by distributing them among five bonding, two non-bonding, and five antibonding $\pi$ molecular orbitals (MOs). These calculations were carried out with the aug-cc-pVDZ basis set. $^{21,22}$

In order to include the effects of dynamic electron correlation, ${ }^{23}(12 / 12)$ CASPT2 calculations ${ }^{24}$ were performed, and the CASSCF geometries were reoptimized at this level of theory. For these calculations the aug-cc-pVDZ basis set was also used. Additional calculations with the aug-cc-pVTZ showed that basis-set expansion had a negligible effect on the computed, relative energies of the electronic states. ${ }^{13}$

All of the CASSCF and CASPT2 calculations were performed with the MOLCAS (version 8.0) suite of programs. ${ }^{25}$ The fully optimized CASSCF and CASPT2 geometries and the CASSCF zero-point energies are available in the Supporting Information for this manuscript.

Simulations of the vibrational structures in the NIPE spectra, based on Franck-Condon Factors (FCFs), were performed using the ezSpectrum (version 3.0) program developed by Mozhayskiy and Krylov. ${ }^{26}$ The necessary equilibrium geometries, harmonic vibrational frequencies, and normal mode vectors for $\mathbf{N Q}^{\mathbf{0}^{-}}$and NQ were obtained from the CASPT2/augcc-pVDZ calculations. 


\section{Results and Discussion}

\section{The NIPE Spectra of NQ ${ }^{-}$}

The NIPE spectra of NQ ${ }^{--}$, obtained with 193, 240, 266, 300, and $355 \mathrm{~nm}$ photons, are shown in Figures 4(a), (b), (c), (d), and (e), respectively. Spectrum 4a, obtained with $193 \mathrm{~nm}$ photons, shows a first band with an electron binding energy (EBE) of 2.8 to $3.3 \mathrm{eV}$, followed by a series of complex bands, until the detachment threshold at $6.4 \mathrm{eV}$ is reached. The first two bands are marked as $\mathrm{X}$, and $\mathrm{A}$. The intensity of band $\mathrm{X}$ is higher than that of band $\mathrm{A}$ in the 193 nm spectrum. 


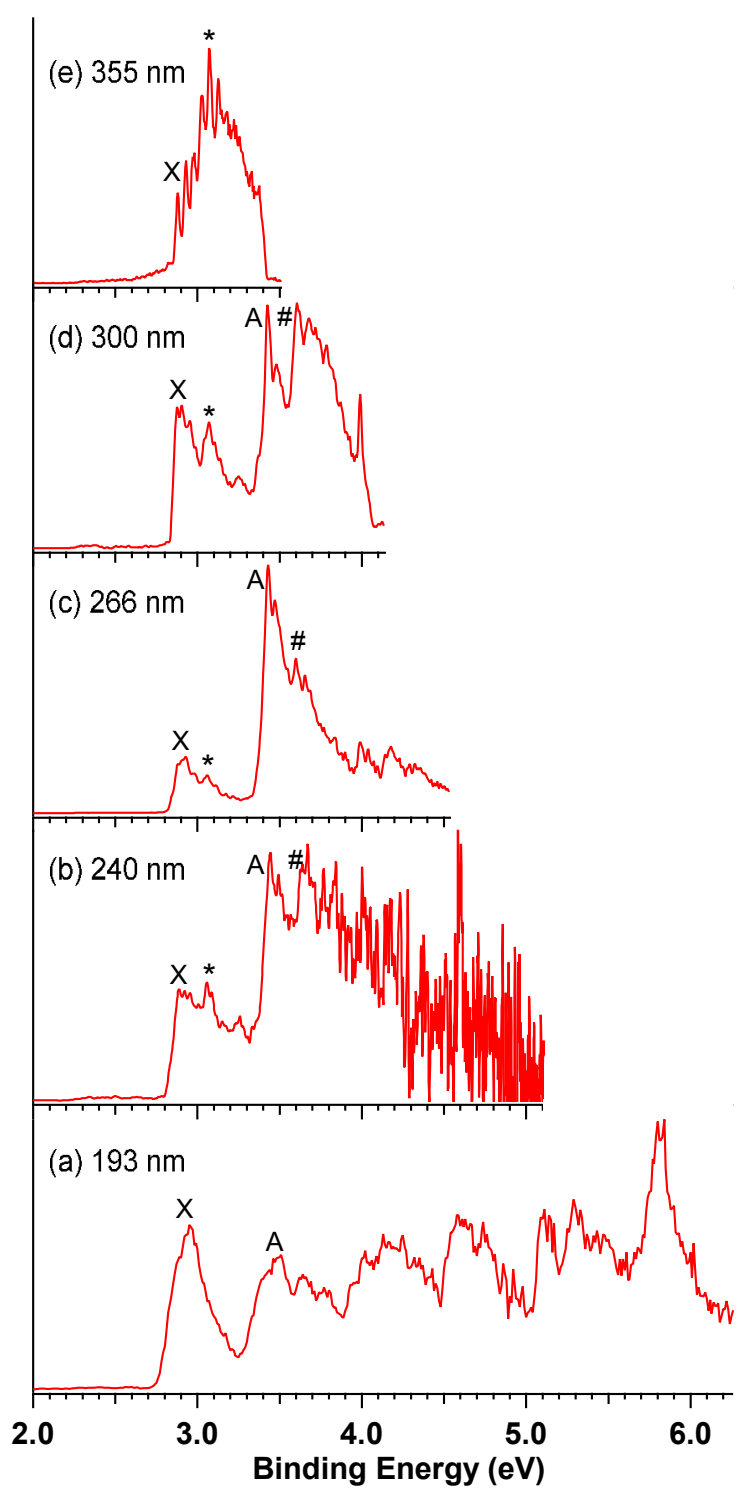

Figure 4. The $20 \mathrm{~K}$ NIPE spectra of $\mathbf{N Q}^{--}$at (a) 193, (b) 240, (c) 266, (d) 300, and (e) $355 \mathrm{~nm}$. The origins of the bands for what appear to be the first two electronic states of NQ are marked X and A. Bands * and \# are most likely due to resonant autodetachment (AD) (see text for details).

In the $240 \mathrm{~nm}$ spectrum (Figure 4b), the intensity of band A has increased dramatically, making it the highest peak. An additional small peak starts to show up between band X and A, at $\sim 3.06 \mathrm{eV}$, and is marked with an asterisk $(*)$. The spectrum intensity on the higher binding energy side of band * increases with it and fills the gap between the $\mathrm{X}$ and $\mathrm{A}$ bands. The peak at 
$\sim 3.63 \mathrm{eV}$ also gains more intensity, and is marked with a hash (\#). The EBEs of the origins of band $\mathrm{X}$ and $\mathrm{A}$ in the $240 \mathrm{~nm}$ spectrum are measured to be 2.88 and $3.43 \mathrm{eV}$, respectively.

At $266 \mathrm{~nm}$, the intensity of band A has increased even more significantly (Figure 4c). The intensities of both peaks at $\sim 3.06 \mathrm{eV}$ and $3.6 \mathrm{eV}$, however, become lower. The EBE of the first peak of band \# decreases from $3.63 \mathrm{eV}$ at $240 \mathrm{~nm}$ to $3.60 \mathrm{eV}$ at $266 \mathrm{~nm}$.

In the $300 \mathrm{~nm}$ spectrum in Figure 4d, band X, band *, band A, and band \# show similar relative intensities as at $240 \mathrm{~nm}$. Band A is stronger than band X. Bands * and \# show stronger intensity than in the $266 \mathrm{~nm}$ spectrum. The peak in band * at $3.06 \mathrm{eV}$ has become only slightly lower than band $\mathrm{X}$; band \#, located at $\mathrm{EBE}=3.605 \mathrm{eV}$, has become so strong that it has equal height as band A.

The $355 \mathrm{~nm}$ spectrum in Figure 4e has the highest energy resolution, and shows some clearly resolved vibrational peaks. The feature at $\mathrm{EBE}=3.07 \mathrm{eV}$ has become the strongest peak in the spectrum, and the envelope of band $\mathrm{X}$ has completely changed.

Taken together, the five NIPE spectra displayed in Figure 4 show that the relative intensities of bands $\mathrm{X}, \mathrm{A},{ }^{*}$, and \# vary considerably at different detachment photon energies. There is no monotonic relationship between detachment photon energy and the peak intensity. The spectral feature at $E B E \approx 3.06 \mathrm{eV}$, first appears with similar intensity as $\mathrm{X}$ in the $240 \mathrm{~nm}$ spectrum (Figure 4b), then becomes weaker in the $266 \mathrm{~nm}$ spectrum, and stronger again at 300 $\mathrm{nm}$. In the spectrum at $355 \mathrm{~nm}$, in Figure 4e, this peak becomes the dominant one. Resonant autodetachment (AD) may contribute to the bands at $E B E \sim 3.06$ and $\sim 3.6 \mathrm{eV}$, and it will be discussed later.

\section{Calculated NIPE Spectrum and the Vibrational Progressions in It}




\section{Geometries and Energies of the Electronic States of $\mathrm{NQ}^{\circ-}$ and NQ}

The CASPT2 geometries of the two lowest electronic states of $\mathbf{N Q}^{-}$and the three lowest electronic states of $\mathbf{N Q}$ are given in Table 1, and their relative energies are provided in Table 2. The CASPT2 results for NQ have been previously published ${ }^{13}$ and are given here for comparison with those for $\mathbf{N Q}^{\cdot-}$. In Table 2 the relative CASPT2 energies are also compared with the experimental values, obtained from the NIPE spectra.

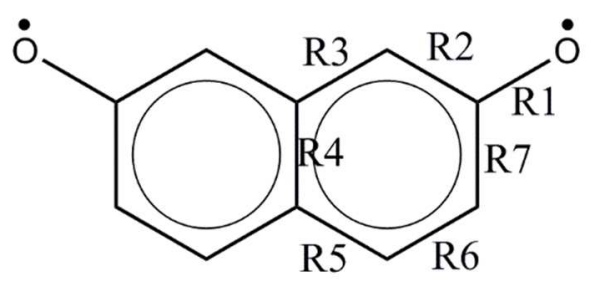

Figure 5. Definitions of the bond lengths in NQ.

Table 1. (12/12)CASPT2 C-C and C-O bond lengths $(\AA)$ in the ${ }^{2} \mathrm{~A}_{2}$ and ${ }^{2} \mathrm{~B}_{1}$ states of $\mathbf{N Q}^{--}$and the ${ }^{3} \mathrm{~B}_{2},{ }^{1} \mathrm{~A}_{1}$, and ${ }^{1} \mathrm{~B}_{2}$ states of $\mathbf{N Q}$, optimized using the aug-cc-pVDZ basis set. The bond lengths are defined in Figure 5.

\begin{tabular}{c|ccccccc}
\hline State & $\mathrm{R} 1$ & $\mathrm{R} 2$ & $\mathrm{R} 3$ & $\mathrm{R} 4$ & $\mathrm{R} 5$ & $\mathrm{R} 6$ & $\mathrm{R} 7$ \\
\hline${ }^{2} \mathrm{~A}_{2}$ & 1.273 & 1.457 & 1.423 & 1.438 & 1.426 & 1.391 & 1.455 \\
${ }^{2} \mathrm{~B}_{1}$ & 1.279 & 1.426 & 1.422 & 1.464 & 1.432 & 1.380 & 1.479 \\
${ }^{3} \mathrm{~B}_{2}$ & 1.255 & 1.456 & 1.416 & 1.453 & 1.434 & 1.379 & 1.470 \\
${ }^{1} \mathrm{~A}_{1}$ & 1.269 & 1.439 & 1.434 & 1.415 & 1.445 & 1.374 & 1.465 \\
${ }^{1} \mathrm{~B}_{2}$ & 1.242 & 1.476 & 1.407 & 1.490 & 1.421 & 1.389 & 1.476 \\
\hline
\end{tabular}


As shown in Table $2, \mathbf{N Q}^{--}$is calculated to have $\mathrm{a}^{2} \mathrm{~A}_{2}$ ground state, but with a ${ }^{2} \mathrm{~B}_{1}$ excited state lying only $0.28 \mathrm{eV}$ above it. In the former state of $\mathbf{N Q}^{--}$the $\mathrm{a}_{2} \pi$ non-bonding (NB) $\mathrm{MO}$ is singly occupied, and the $b_{1} \pi$ NBMO is doubly occupied; and in the latter state the $a_{2}$ NBMO is doubly occupied and the $b_{1}$ NBMO is singly occupied. Removal of the appropriate electron from the doubly occupied NBMO in either state of $\mathbf{N Q}^{\bullet-}$ gives rise to the ${ }^{3} \mathrm{~B}_{2}$ ground state of NQ. The $a_{2}$ and $b_{1}$ NBMOs of the ${ }^{3} B_{2}$ state are shown in Figure 6 .

Table 2. CASPT2/aug-cc-pVDZ relative energies $(\Delta E$ in $\mathrm{eV})$ of the low-lying electronic states of $\mathbf{N Q}^{--}$and NQ, including CASSCF/aug-cc-pVDZ zero-point energy corrections. The assignments of the three major peaks in the NIPE spectrum of $\mathbf{N Q}^{--}$and the experimental electron binding energy (EBE) $(\mathrm{eV})$ of each are given for comparison with the CASPT2 energies. Relative energies of the low-lying electronic states, with reference to the ground state of NQ ( $\Delta E^{\prime}$ in $\left.\mathrm{kcal} / \mathrm{mol}\right)$ are also presented.

\begin{tabular}{lccccc}
\hline & ${ }^{2} \mathrm{~A}_{2}$ & ${ }^{2} \mathrm{~B}_{1}$ & ${ }^{3} \mathrm{~B}_{2}$ & ${ }^{1} \mathrm{~B}_{2}$ & ${ }^{1} \mathrm{~A}_{1}$ \\
\hline Wave function & $\mid \mathrm{b}_{1}{ }^{2} \mathrm{a}_{2}>$ & $\mid \mathrm{b}_{1} \mathrm{a}_{2}{ }^{2}>$ & $\mid \mathrm{b}_{1} \mathrm{a}_{2}(\alpha \beta+\beta \alpha)$ & $\mid \mathrm{b}_{1} \mathrm{a}_{2}(\alpha \beta-\beta \alpha)>$ & $\left|\mathrm{b}_{1}{ }^{2}>-\right| \mathrm{a}_{2}{ }^{2}>$ \\
$\Delta E($ CASPT2, eV) & 0 & 0.28 & 2.98 & 3.45 & 3.53 \\
NIPES Peak, $E B E(\mathrm{eV})$ & & & $\mathrm{X}, 2.88(1)$ & $\mathrm{A}, 3.43(1)$ & 3.59 \\
$\Delta E^{\prime}(\mathrm{CASPT}, \mathrm{kcal} / \mathrm{mol})$ & & & 11.0 & 12.9 \\
$\Delta E^{\prime}$ (NIPES, kcal/mol) & & & 12.7 & 16.4 \\
\hline
\end{tabular}

${ }^{\mathrm{a}}$ The numbers in parentheses represent experimental uncertainty in the last digits. The $E B E=3.59$ $\mathrm{eV}$ for ${ }^{1} \mathrm{~A}_{1}$ is an estimated value. 


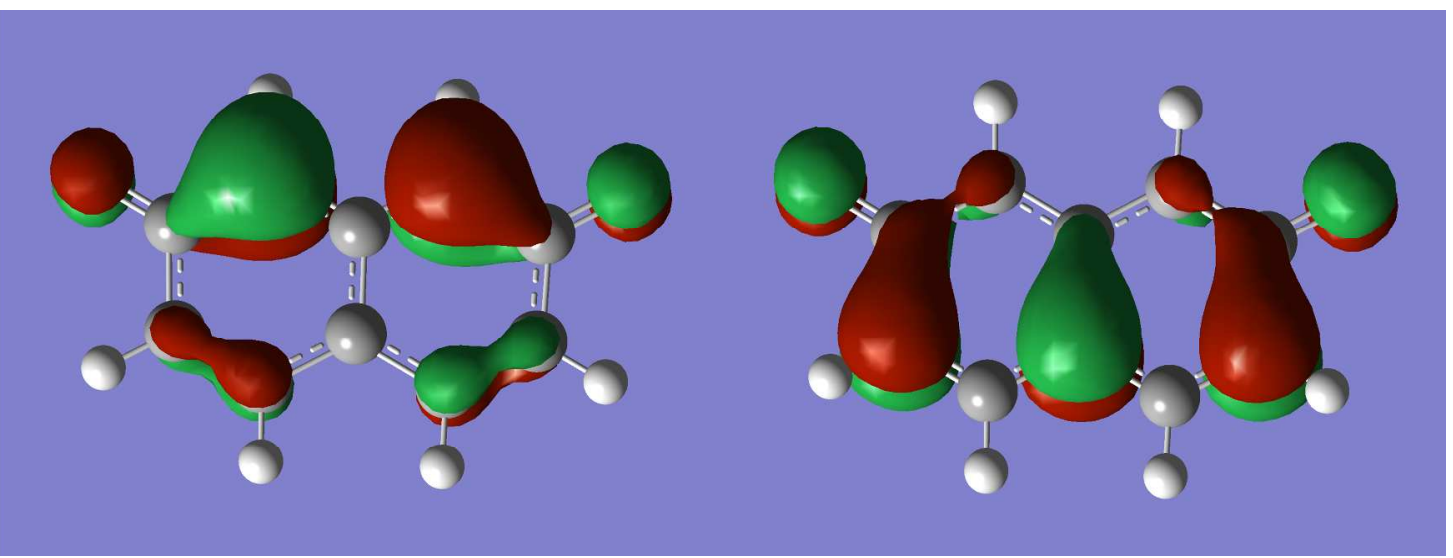

Figure 6. The $a_{2}$ and $b_{1}$ NBMOs of the ${ }^{3} B_{2}$ ground state of NQ

\section{Assignment of the NIPE Spectra}

The calculated CASPT2/aug-cc-pVDZ energy of the ${ }^{3} \mathrm{~B}_{2}$ state of $\mathbf{N Q}$ of $2.98 \mathrm{eV}$, relative to the ${ }^{2} \mathrm{~A}_{2}$ ground state of $\mathbf{N Q}^{--}$, is in good agreement with the experimental value of $E B E=$ $2.88 \mathrm{eV}$ of band $\mathrm{X}$ in the NIPE spectrum. However, due to the appearance of the features * and \# and the considerable change in the relative peak intensities at different energies of the detaching photons, the assignment of the peaks in the NIPE spectrum is not straightforward. In order to obtain a convincing assignment of the peaks, the origins of features * and \# need to be explained.

The $355 \mathrm{~nm}$ NIPE spectrum shows a series of well resolved peaks, with the predominant peak being band *, which is located at $E B E=3.07 \mathrm{eV}$. Does the lower intensity of band $\mathrm{X}$, relative to band *, in the $355 \mathrm{~nm}$ NIPE spectrum come from band $\mathrm{X}$ being a hot band? An experiment that assessed the temperature dependence of the $355 \mathrm{~nm}$ NIPE spectrum was performed, in order to test this possibility. The NIPE spectra with $355 \mathrm{~nm}$ photons at both room temperature $(303 \mathrm{~K})$ and at low temperature $(20 \mathrm{~K})$ were taken and plotted together for comparison. (See Figure S4 in the Supporting Information). Comparison of these two spectra indicates that hot bands do, indeed, exist at $303 \mathrm{~K}$; but they only contribute to the threshold 
region between 2.8 to $2.85 \mathrm{eV}$ below the energy of band $\mathrm{X}$. The resolved peaks of band $\mathrm{X}$ at 355 $\mathrm{nm}$ in spectrum (e) of Figure 4 are not due to hot bands.

Photodetaching fragments are another possible reason for the appearance of peaks * and \# in the NIPE spectra, and this possibility was checked by experiments with different photon fluxes. NIPE spectra of NQ $^{\circ-}$ with $266 \mathrm{~nm}$ photons were taken at photon fluxes of $0.01 \mathrm{~mJ} / \mathrm{pulse}$ and $0.11 \mathrm{~mJ} /$ pulse (Figure S5). If peaks * and \# were due to detachment of daughter ions, formed by photodissociation of the parent anion by the first photon, peaks * and \# would be formed by a process involving two photons. Therefore, significant intensity changes in the $266 \mathrm{~nm}$ NIPE spectrum should be observed as the photon flux is changed an order of magnitude. However, Figure S5 shows that essentially same spectrum was obtained for the two different photon fluxes. This observation indicates that the appearance of the NIPE spectrum is overwhelmingly dictated by a one-photon process, and it thus rules out the possibility of a photodetaching fragment process.

\section{Resonant Autodetachment and Direct Electron Detachment in NQ ${ }^{--}$}

Based on the above experimental evidence, which rules out hot bands and two-photon processes as being responsible for bands * and \# in the NIPE spectrum in Figure 4, we believe that the most likely explanation of their origin is that they come from resonant autodetachment in $\mathbf{N Q}^{-}$. Resonant autodetachment from an anion occurs by a pathway that is different from formation of the corresponding neutral molecule by direct electron loss. In resonant autodetachment, photoexcitation leads to creation of an excited electronic state of the anion, from which electron loss subsequently occurs. Such a pathway gives 'extra' bands in NIPE spectra, bands that are higher in energy than the threshold for direct electron loss. The contribution of these extra bands to a NIPE spectrum depends on the energy of the laser that is 
used for electron photodetachment, since resonant autodetachment bands are strongest when the laser energy matches the energy of an excited state from which autodetachment occurs.

Such 'extra' bands, due to resonant autodetachment, have been observed before in NIPE spectra. For example, the $266 \mathrm{~nm}$ NIPE spectrum of $\mathrm{C}_{60}{ }^{-}$shows an extra band at 3.7-4.0 eV, a region that is supposed to be "clean", because it lies between the energy of the singlet and triplet state of $\mathrm{C}_{60}{ }^{27}$ Autodetachment from resonant excited states is commonly observed in the anions of aromatic organic molecules. Weinkauf and Schiedt studied resonant autodetachment from the anion of $p$-benzoquinone at $0.15-0.65 \mathrm{eV}$ above the direct detachment threshold, and they showed that the resonant autodetachment, not direct excitation into the continuum, is responsible for the bands in this region. ${ }^{28}$ Lineberger and co-workers found the appearance of autodetachment peaks at a laser wavelength of $351 \mathrm{~nm}$ and disappearance of those peaks at a laser wavelength of $364 \mathrm{~nm}$ in the NIPES study of phenoxide. ${ }^{29}$ Very recently, Verlet et al. studied resonant autodetachment in the anthracenyl anion, and they showed that a number of $\pi^{*}$ resonances are active in leading to electron loss in the first $2.5 \mathrm{eV}$ above the threshold. ${ }^{30}$

We believe that in the $\mathbf{N Q}^{--}$radical anion, electronic transitions from the ${ }^{2} \mathrm{~A}_{2}$ ground state to certain excited states of the anion, followed by electron loss, give rise to the autodetachment bands at $E B E=3.06 \mathrm{eV}(*)$ and $E B E=3.63 \mathrm{eV}(\#)$ in the NIPE spectra in Figure 4. However, we assign bands $\mathrm{X}$ and $\mathrm{A}$ in the NIPE spectrum of $\mathbf{N Q}^{--}$to direct electron detachment from the ground state of the radical anion to, respectively, the ground state and a low-lying excited state of neutral NQ.

On the basis of good agreement in Table 2 between the CASPT2 EBE of the ${ }^{3} \mathrm{~B}_{2}$ state of NQ and the $E B E$ measured for peak $\mathrm{X}$ in the NIPE spectrum of $\mathbf{N Q}^{-}$, we can confidently assign band $\mathrm{X}$ in the NIPE spectra to the direct transition from the ${ }^{2} \mathrm{~A}_{2}$ ground state of $\mathrm{NQ}^{--}$to the ${ }^{3} \mathrm{~B}_{2}$ 
ground state of neutral NQ. Band A is thus, presumably, due to formation of an excited singlet electronic state of NQ. We tentatively assign band A to formation of the ${ }^{1} \mathrm{~B}_{2}$ state, since it is calculated to be lower in energy than the ${ }^{1} \mathrm{~A}_{1}$ state.

However, the calculated CASPT2 energy difference between ${ }^{1} \mathrm{~B}_{2}$ and ${ }^{1} \mathrm{~A}_{1}$ is small, amounting to only $1.9 \mathrm{kcal} / \mathrm{mol}$. Therefore, we performed Franck-Condon (FC) simulations of the NIPE spectra of $\mathbf{N Q}^{\bullet-}$, in order to make a more convincing assignment of ${ }^{1} \mathrm{~B}_{2}$ or ${ }^{1} \mathrm{~A}_{1}$ as being responsible for peak $\mathrm{A}$ in the experimental spectrum.

\section{Franck-Condon Simulation of the NIPE Spectra}

The FC simulations were performed with the ezSpectrum program, using the CASPT2/augcc-pVDZ optimized geometry, vibrational frequencies, and normal mode vectors for each electronic state. The ${ }^{2} \mathrm{~A}_{2}$ ground state of $\mathbf{N Q}^{\bullet-}$ was taken as the initial state, and the three, lowlying states of $\mathbf{N Q}-{ }^{3} \mathrm{~B}_{2},{ }^{1} \mathrm{~B}_{2}$, and ${ }^{1} \mathrm{~A}_{1}-$ were each taken as the final state. The results of the $\mathrm{FC}$ simulations are shown in Figure 7.

In Figure 7 the energy and the intensity of the $0-0$ peak in the vibrational progression computed for each state have been adjusted to match those in experimental spectrum. The fullwidth-half-maxima (FWHM) of the simulated peaks were set to match the experimental resolution. Because the experiment was performed at low temperature $(20 \mathrm{~K})$, no peaks from hot bands appeared in the FC simulations.

The spectra obtained with $355 \mathrm{~nm}$ photons show the strongest * peak. Consequently the resonant autodetachment that gives rise to this peak resonance is likely to alter the intensities of vibrational bands in the nearby X peak. Therefore, only the spectra obtained with $240 \mathrm{~nm}, 266$ $\mathrm{nm}$, and $300 \mathrm{~nm}$ photons are used in Figure 7 for comparison with the FC simulations. The 
comparison of the $355 \mathrm{~nm}$ spectrum with the FC simulations of it and the assignment of the observed peaks in this spectrum are given in the Supporting Information.

On the basis of the comparison of the calculated EBEs and FC intensities, with those in the experimental NIPE spectrum, the 0-0 transitions within bands X and A can be confidently assigned to the ${ }^{3} \mathrm{~B}_{2}$ and ${ }^{1} \mathrm{~B}_{2}$ states respectively, giving experimental values of $E B E=2.88 \mathrm{eV}$ for the triplet and $E B E=3.43 \mathrm{eV}$ for the lowest singlet state. However, the assignment of the $0-0$ transition in ${ }^{1} \mathrm{~A}_{1}$ is more difficult, due to the complexity of the spectra and the size of the gap between ${ }^{1} \mathrm{~B}_{2}$ and ${ }^{1} \mathrm{~A}_{1}$ states, which is calculated to be small. The peak for the $0-0$ band of the ${ }^{1} \mathrm{~A}_{1}$ state is probably buried under the A and \# bands.

By carefully comparing the NIPE spectra at different photon energies, we find some clues about the location of the $0-0$ peak for ${ }^{1} \mathrm{~A}_{1}$ from the difference in the appearance of the \# band. The $E B E$ of the first small peak of the \# band is $3.63 \mathrm{eV}$ in the $240 \mathrm{~nm}$ spectrum, but $3.60 \mathrm{eV}$ in the $266 \mathrm{~nm}$ spectrum, and $3.605 \mathrm{eV}$ in the $300 \mathrm{~nm}$ spectrum. The $\sim 30 \mathrm{meV} E B E$ difference may be caused by the presence of the ${ }^{1} \mathrm{~A}_{1}$ state. By carefully moving the ${ }^{1} \mathrm{~A}_{1}$ state location, changing its intensity, and comparing the experimental spectrum with the simulated spectrum, the $0-0$ transition of ${ }^{1} \mathrm{~A}_{1}$ has been provisionally located at $3.59 \mathrm{eV}$.

However, the assignment of the $E B E$ of ${ }^{1} \mathrm{~A}_{1}$ is challenging. For example, if the ${ }^{1} \mathrm{~A}_{1}$ peak is shifted closer to the ${ }^{1} \mathrm{~B}_{2}$ peak, reducing the gap between the two states to $2.7 \mathrm{kcal} / \mathrm{mol}$, the simulated spectrum could also fit the experimental spectrum at a similar level of quality as in Figure 7. (see Figure $\mathrm{S} 1$ in the $\mathrm{SI}$ ). However, $E B E=3.59 \mathrm{eV}$ is still the best estimation for the position of the $0-0$ transition in the ${ }^{1} \mathrm{~A}_{1}$ state. 


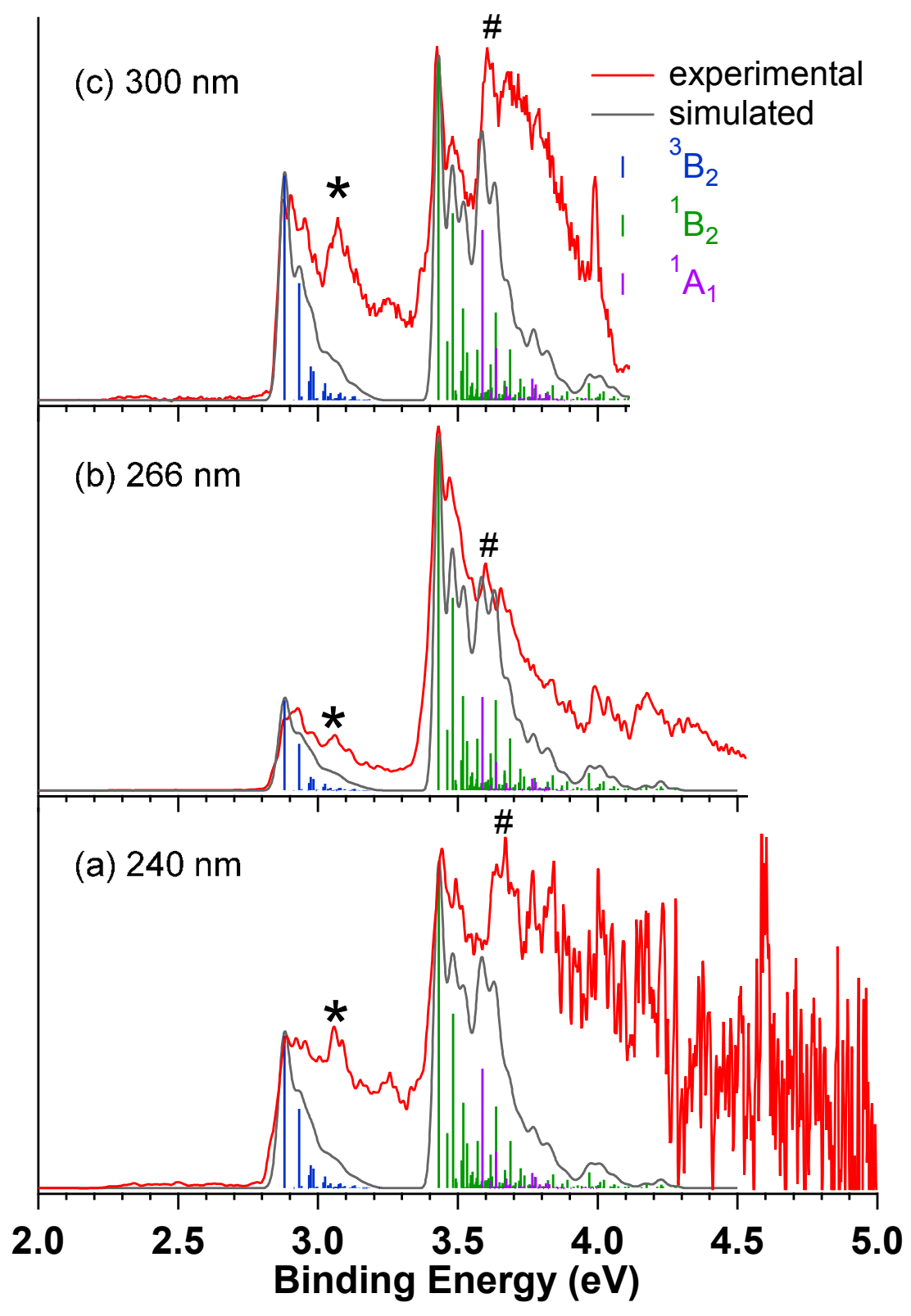

Figure 7. Simulated NIPE spectra for formation of the three lowest electronic states of NQ, using the calculated CASPT2/aug-cc-pVDZ stick spectra, convoluted with Gaussian line broadening. The simulated spectra are superimposed onto the experimental spectra of $\mathbf{N Q}^{-}$at (a) $240 \mathrm{~nm}$, (b) $266 \mathrm{~nm}$, and (c) $300 \mathrm{~nm}$. The simulated peak intensities and positions of the 0-0 bands have been adjusted to match those in the experimental spectrum. The FWHMs of Gaussians for ${ }^{3} \mathrm{~B}_{2},{ }^{1} \mathrm{~B}_{2}$, and ${ }^{1} \mathrm{~A}_{1}$ peaks are set to $60,50,50 \mathrm{meV}$ at $240 \mathrm{~nm} ; 50,35,35 \mathrm{meV}$ at 266 $\mathrm{nm}$; and $45,35,35 \mathrm{meV}$ at $300 \mathrm{~nm}$, respectively. The energy difference between ${ }^{1} \mathrm{~B}_{2}$ and ${ }^{1} \mathrm{~A}_{1}$ is set to $3.7 \mathrm{kcal} / \mathrm{mol}$. 
The simulated spectra with $240 \mathrm{~nm}, 266 \mathrm{~nm}$, and $300 \mathrm{~nm}$ photons are displayed in Figure 7, together with the experimental NIPE spectra for comparison. The CASPT2/aug-cc-pVDZ simulated spectrum at $193 \mathrm{~nm}$, and more simulations using different theoretical methods [i.e., (12/12)CASSCF and B3LYP], are given in the SI of this manuscript. Overall, the simulated NIPE spectra provide a good fit to the experimental spectra, except for the peaks * and \#, which we believe are due to resonant autodetachment. The energies, derived from the NIPE spectrum, of the two singlet states of NQ, relative to the triplet ground state, are listed in Table 2. The experimental value of $\Delta E_{\mathrm{ST}}$ is estimated to be $0.55 \mathrm{eV}=12.7 \mathrm{kcal} / \mathrm{mol}$. The previously calculated value of the energy difference between ${ }^{3} \mathrm{~B}_{2}$ and ${ }^{1} \mathrm{~B}_{2}$ states of $\Delta E_{\mathrm{ST}}=11.0 \mathrm{kcal} / \mathrm{mol}$ agrees well with this experimental value.

The experimental $E B E$ of the ${ }^{1} \mathrm{~A}_{1}$ state is, as discussed above, less reliable than the EBEs of the ${ }^{3} \mathrm{~B}_{2}$ and ${ }^{1} \mathrm{~B}_{2}$ states. The $E B E$ of ${ }^{1} \mathrm{~A}_{1}$ is estimated to be $3.59 \mathrm{eV}$, which is $0.71 \mathrm{eV}(16.4$ $\mathrm{kcal} / \mathrm{mol})$ higher than that of the ${ }^{3} \mathrm{~B}_{2}$ ground state and $0.16 \mathrm{eV}(3.7 \mathrm{kcal} / \mathrm{mol})$ higher than that of the ${ }^{1} \mathrm{~B}_{2}$ state. The calculated CASPT2 energy differences are, respectively, $12.9 \mathrm{kcal} / \mathrm{mol}$ and 1.9 $\mathrm{kcal} / \mathrm{mol}$.

As shown in Figure 7, the FC simulations find that, of the two NQ singlet states, the open-shell singlet ${ }^{1} \mathrm{~B}_{2}$ state is calculated to have the longer vibrational progression. It is easy to see that the green ${ }^{1} \mathrm{~B}_{2}$ peaks extend over a much wider binding energy range than the purple ${ }^{1} \mathrm{~A}_{1}$ peaks. The reason is that, as shown in Table 1 , the difference in geometry between the ${ }^{1} \mathrm{~B}_{2}$ state of NQ and the ${ }^{2} \mathrm{~A}_{2}$ state of $\mathbf{N Q}^{--}$is larger than the difference between ${ }^{1} \mathrm{~A}_{1}$ and ${ }^{2} \mathrm{~A}_{2}$; and large changes in geometry between radical anion and neutral give rise to long vibrational progressions. 
In particular, as shown in Table 1, the $\mathrm{C}=\mathrm{O}$ bond lengths ( $\mathrm{R} 1$ ) are calculated to be 0.027 $\AA$ shorter in ${ }^{1} \mathrm{~B}_{2}$ than in ${ }^{1} \mathrm{~A}_{1}$. The greater change in the $\mathrm{C}=\mathrm{O}$ bond lengths should result in a longer progression in the symmetrical $\mathrm{C}=\mathrm{O}$ stretching mode in the peaks for formation of ${ }^{1} \mathrm{~B}_{2}$ than of ${ }^{1} \mathrm{~A}_{1}$. This predicted difference between the two singlet states provides, at least in principle, a way to distinguish between them in the NIPE spectrum of $\mathbf{N Q}^{-}$.

\section{Summary and Conclusions}

NIPE spectra of $\mathbf{N Q}^{-}$have been taken at $20 \mathrm{~K}$, using 193, 240, 266, 300, and $355 \mathrm{~nm}$ lasers for electron photodetachment. CASPT2/aug-cc-pVDZ calculations predict with reasonable accuracy the $E B E$ s of the $0-0$ bands in the three lowest electronic states of NQ. In addition, the FCFs, calculated from the CASPT2/aug-cc-pVDZ optimized geometries, vibrational frequencies, and normal modes, successfully simulate the appearance of the first two of these three bands.

The following assignments of the $E B E$ s of the electronic states of NQ in the NIPE spectrum of $\mathbf{N Q}^{--}$have been made on the basis of the simulations of the NIPE spectra in Figure 7. Peak $\mathrm{X}$ at $E B E=2.88 \mathrm{eV}$ is the origin of the ${ }^{3} \mathrm{~B}_{2}$ state; peak $\mathrm{A}$ at $E B E=3.43 \mathrm{eV}$ is the origin of the ${ }^{1} \mathrm{~B}_{2}$ state; and the origin of the ${ }^{1} \mathrm{~A}_{1}$ state is estimated to have $E B E=3.59 \mathrm{eV}$. These assignments of the bands in the NIPE spectrum confirm that, as predicted, ${ }^{13} \mathrm{NQ}$ has a ${ }^{3} \mathrm{~B}_{2}$ triplet ground state, and the energy of the ${ }^{1} \mathrm{~B}_{2}$ state is slightly lower than that of the ${ }^{1} \mathrm{~A}_{1}$ state. NIPES provides an experimental value of $\Delta E_{\mathrm{ST}}=12.7 \mathrm{kcal} / \mathrm{mol}$ in $\mathrm{NQ}$, about $1.7 \mathrm{kcal} / \mathrm{mol}$ larger than the CASPT2 calculated value of $\Delta E_{\mathrm{ST}}=11.0 \mathrm{kcal} / \mathrm{mol},{ }^{13}$ and the ${ }^{1} \mathrm{~B}_{2}-{ }^{1} \mathrm{~A}_{1}$ gap $=3.7 \mathrm{kcal} / \mathrm{mol}$, which is about $1.8 \mathrm{kcal} / \mathrm{mol}$ larger than the calculated value.

The experimental NIPE spectrum of $\mathbf{N Q}^{--}$gives not only the same state ordering as that predicted by the calculations in Reference 13, but also energetic separations between the states 
that are similar to those predicted by these calculations. Consequently, the experimental NIPE spectrum of $\mathbf{N Q}^{--}$provides evidence, albeit circumstantial, that these calculations are likely also to have also predicted correctly the still experimentally unknown NIPE spectrum of NQDM- .

Obtaining the latter NIPE spectrum is, of course, necessary, in order to fully test the prediction that the substitution of both methylene groups in NQDM by the oxygens in NQ will be found to increase $\Delta E_{\mathrm{ST}}$, rather than to decrease $\Delta E_{\mathrm{ST}}$ by $17.5 \mathrm{kcal} / \mathrm{mol}$, as has been found to be the case for TMM $\rightarrow \mathbf{O X A},{ }^{7,8}$ or to leave $\Delta E_{\mathrm{ST}}$ almost unchanged, as has been found to be the case for MQBDM $\rightarrow$ MBQ. ${ }^{9-11}$

Supporting Information. (12/12)CASSCF and CASPT2 optimized geometries, energies, frequencies, and thermal corrections for the low-lying states of NQ and NQ ${ }^{--}$; Simulated NIPE spectrum with a different ${ }^{1} \mathrm{~B}_{2}$ and ${ }^{1} \mathrm{~A}_{1}$ separation $(2.7 \mathrm{kacl} / \mathrm{mol})$; Simulated NIPE spectra using different levels of theory, i.e., (12/12)CASPT2, (12/12)CASSCF, and B3LYP; Simulated $193 \mathrm{~nm}$ and $355 \mathrm{~nm}$ spectrum and assignment of the vibrational peaks in the $355 \mathrm{~nm}$ spectrum; Comparison of the hot $(303 \mathrm{~K})$ and cold $(20 \mathrm{~K}) 355 \mathrm{~nm}$ spectrum; Comparison of the $266 \mathrm{~nm}$ spectra with different photon fluxes.

\section{Author Information}

Corresponding Authors

Weston T. Borden: Weston.Borden@unt.edu

Xue-Bin Wang: Xuebin.wang@pnnl.gov

\section{Notes}

The authors declare no competing financial interest 
Acknowledgement. W. T. B. acknowledges the generous support of his research by Grant B0027 from the Robert A. Welch Foundation. The photoelectron spectroscopy work done at PNNL was supported by U.S. Department of Energy (DOE), Office of Science, Office of Basic Energy Sciences, Division of Chemical Sciences, Geosciences, and Biosciences (XBW), and performed in EMSL, a national scientific user facility sponsored by DOE's Office of Biological and Environmental Research and located at Pacific Northwest National Laboratory, which is operated by Battelle Memorial Institute for the DOE.

\section{References and Notes}

1. Osamura, Y.; Borden, W. T.; Morokuma, K. Structure and Stability of Oxyallyl. An MCSCF Study. J. Am. Chem. Soc. 1984, 106, 5112-5115.

2. Coolidge, M. B.; Yamashita, K.; Morokuma, K.; Borden, W. T. Ab initio MCSCF and CI Calculations of the Singlet-triplet Energy Differences in Oxyallyl and in Dimethyloxyallyl. $J$. Am. Chem. Soc. 1990, 112, 1751-1754.

3. Ervin, K. M. ; Lineberger, W. C. In Advances in Gas Phase Ion Chemistry, Adams, N. G.; Babcock, L.M. Ed. JAI Press: Greenwich, 1992; Vol. 1, pp 121-166.

4. Lineberger, W. C.; Borden, W. T. The Synergy between Qualitative Theory, Quantitative Calculations, and Direct Experiments in Understanding, Calculating, and Measuring the Energy Differences between the Lowest Singlet and Triplet States of Organic Diradicals. Phys. Chem. Chem. Phys. 2011, 13, 11792-11813.

5. Wenthold, P. G.; Hu, J.; Squires, R. R.; Lineberger, W. C., Photoelectron Spectroscopy of the Trimethylenemethane Negative Ion. The Singlet-Triplet Splitting of Trimethylenemethane. J. Am. Chem. Soc. 1996, 118, 475-476.

6. Wenthold, P. G.; Hu, J.; Squires, R. R.; Lineberger, W. C., Photoelectron Spectroscopy of the Trimethylenemethane Negative Ion. J. Am. Soc. Mass Spectrom. 1999, 10, 800-809.

7. Ichino, T.; Villano, S. M.; Gianola, A. J.; Goebbert, D. J.; Velarde, L.; Sanov, A.; Blanksby, S. J.; Zhou, X.; Hrovat, D. A.; Borden, W. T.; et al. The Lowest Singlet and Triplet States of the Oxyallyl Diradical. Angew. Chem. Int. Ed. 2009, 48, 8509-8511.

8. Ichino, T.; Villano, S. M.; Gianola, A. J.; Goebbert, D. J.; Velarde, L.; Sanov, A.; Blanksby, S. J.; Zhou, X.; Hrovat, D. A.; Borden, W. T.; et al. Photoelectron Spectroscopic Study of the Oxyallyl Diradical. J. Phy, Chem. A 2011, 115, 1634-1649.

9. $\quad$ Fort, R. C.; Getty, S. J.; Hrovat, D. A.; Lahti, P. M.; Borden, W. T. Ab initio Calculations on $m$-Quinone. The Ground State Is a Triplet. J. Am. Chem. Soc. 1992, 114, 7549-7552.

10. Chen, B.; Hrovat, D. A.; Deng, S. H. M.; Zhang, J.; Wang, X. B.; Borden, W. T. The Negative Ion Photoelectron Spectrum of meta-Benzoquinone Radical Anion (MBQ ${ }^{-}$): A Joint Experimental and Computational Study. J. Am. Chem. Soc. 2014, 136, 3589-3596. 
11. Fu, Q. A.; Yang, J. L.; Wang, X. B. On the Electronic Structures and Electron Affinities of the m-Benzoquinone (BQ) Diradical and the o-, p-BQ Molecules: A Synergetic Photoelectron Spectroscopic and Theoretical Study. J. Phys. Chem. A 2011, 115, 3201-3207.

12. Wenthold, P. G.; Kim, J. B.; Lineberger, W. C. Photoelectron Spectroscopy of mXylylene Anion. J. Am. Chem. Soc. 1997, 119, 1354-1359.

13. Hrovat, D. A.; Wang, X. B.; Borden, W. T. Calculations of the Relative Energies of the Low-lying Electronic States of 2,7-Naphthoquinodimethane and 2,7-Naphthoquinone.

Substitution of Oxygen for $\mathrm{CH}_{2}$ is Predicted to Increase the Singlet-triplet Energy Difference $\left(\Delta \mathrm{E}_{\mathrm{ST}}\right)$. J. Phys. Org. Chem. 2018; e3824. https://doi.org/10.1002/poc.3824.

14. Gryn'ova, G.; Coote, M. L. Origin and Scope of Long-Range Stabilizing Interactions and Associated SOMO-HOMO Conversion in Distonic Radical Anions. J. Am. Chem. Soc. 2013, $135,15392-15403$.

15. Gryn'ova, G.; Marshall, D. L.; Blanksby, S. J.; Coote, M. L. Switching Radical Stability by $\mathrm{pH}$-Induced Orbital Conversion. Nat. Chem. 2013, 5, 474-481.

16. Wang, X. B.; Wang, L. S. Development of a Low-temperature Photoelectron Spectroscopy Instrument Using an Electrospray Ion Source and a Cryogenically Controlled Ion Trap. Rev. Sci. Instrum. 2008, 79, 073108.

17. Hanstorp, D.; Gustafsson, M. Determination of the Electron Affinity of Iodine. J. Phys. B: At. Mol. Opt. Phys. 1992, 25, 1773-1783.

18. Rienstra-Kiracofe, J. C.; Tschumper, G. S.; Schaefer, H. F.; Nandi, S.; Ellison, G. B. Atomic and Molecular Electron Affinities: Photoelectron Experiments and Theoretical Computations. Chem. Rev. 2002, 102, 231-282.

19. Wang, X. B.; Wang, L. S. Photodetachment of Free Hexahalogenometallate Doubly Charged Anions in the Gas Phase: $\left[\mathrm{ML}_{6}\right]^{2-},(\mathrm{M}=\mathrm{Re}, \mathrm{Os}, \mathrm{Ir}, \mathrm{Pt} ; \mathrm{L}=\mathrm{Cl}$ and $\mathrm{Br})$. J. Chem. Phys. 1999, 111, 4497-4509.

20. Kim, J. B.; Yacovitch, T. I.; Hock, C.; Neumark, D. M. Slow Photoelectron Velocity-map Imaging Spectroscopy of the Phenoxide and Thiophenoxide Anions. Phys. Chem. Chem. Phys. 2011, 13, 17378-17383.

21. Dunning, T. H. Jr. Gaussian Basis Sets for Use in Correlated Molecular Calculations. I. The Atoms Boron through Neon and Hydrogen. J. Chem. Phys. 1989, 90, 1007-1023.

22. Kendall, R. A.; Dunning, T. H.; Harrison, R. J. Electron Affinities of the First-row Atoms Revisited. Systematic Basis Sets and Wave Functions. J. Chem. Phys. 1992, 96, 6796-6806.

23. Borden, W. T.; Davidson, E. R. The Importance of Including Dynamic Electron Correlation in ab Initio Calculations. Acc. Chem. Res. 1996, 29, 67-75.

24. Andersson, K.; Malmqvist, P. Å.; Roos, B. O. Second-order Perturbation Theory with a Complete Active Space Self-consistent Field Reference Function. J. Chem. Phys. 1992, 96, 1218-1226.

25. Aquilante, F.; Autschbach, J.; Carlson, R. K.; Chibotaru, L. F.; Delcey, M. G.; De Vico, L.; Fdez. Galván, I.; Ferré, N.; Frutos, L. M.; Gagliardi, L.; et al. Molcas 8: New Capabilities for Multiconfigurational Quantum Chemical Calculations Across the Periodic Table. J. Comput. Chem. 2016, 37, 506-541.

26. Mozhayskiy, V. A.; Krylov, A. I. ezSpectrum, version 3.0; see http://iopenshell.usc.edu/downloads (accessed April 2017).

27. Wang, X. B.; Ding, C. F.; Wang, L. S. High Resolution Photoelectron Spectroscopy of $\mathrm{C}_{60}{ }^{-}$.J. Chem. Phys. 1999, 110, 8217-8220. 
28. Schiedt, J.; Weinkauf, R. Resonant Photodetachment via Shape and Feshbach Resonances: p-Benzoquinone Anions as a Model System. J. Chem. Phys. 1999, 110, 304-314. 29. Gunion, R. F.; Gilles, M. K.; Polak, M. L.; Lineberger, W. C. Ultraviolet Photoelectron Spectroscopy of the Phenide, Benzyl and Phenoxide Anions, with Ab Initio Calculations. Int. Mass. Spectrom. Ion Processes 1992, 117, 601-620.

30. Stanley, L. H.; Anstoter, C. S.; Verlet, J. R. R. Resonances of the Anthracenyl Anion Probed by Frequency-resolved Photoelectron Imaging of Collision-induced Dissociated Anthracene Carboxylic Acid. Chem. Sci. 2017, 8, 3054-3061. 


\section{TOC graphic}

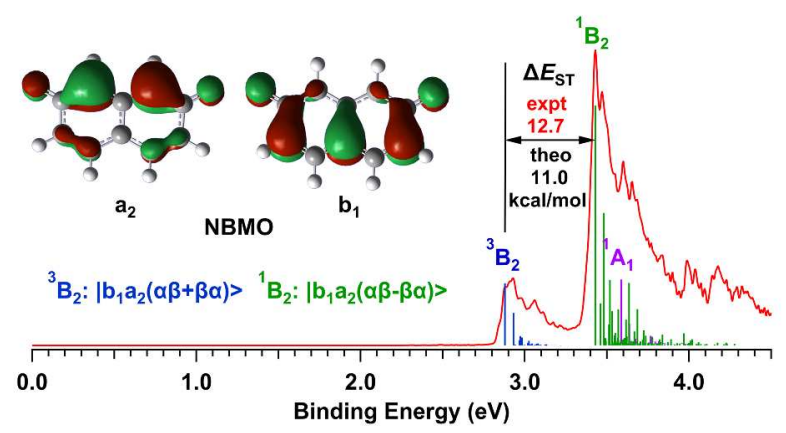

